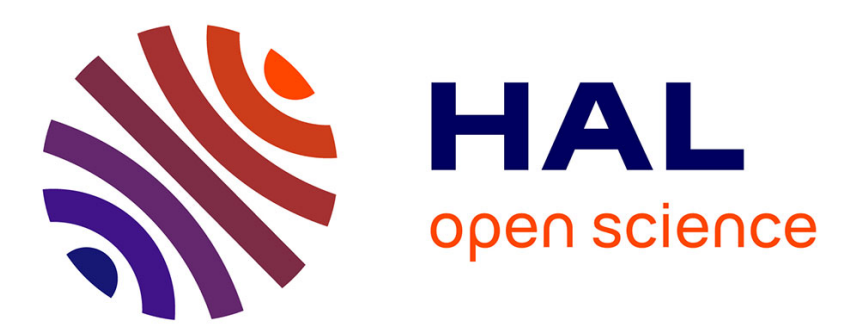

\title{
Global nonlinear dynamics of thin aerofoil wakes
}

Benoît Pier, Nigel Peake

\section{To cite this version:}

Benoît Pier, Nigel Peake. Global nonlinear dynamics of thin aerofoil wakes. Seventh IUTAM Symposium on Laminar-Turbulent Transition, Jun 2009, Stockholm, Sweden. pp.319-324, 10.1007/978-90481-3723-7_51. hal-00633233

\section{HAL Id: hal-00633233 https://hal.science/hal-00633233}

Submitted on 18 Oct 2011

HAL is a multi-disciplinary open access archive for the deposit and dissemination of scientific research documents, whether they are published or not. The documents may come from teaching and research institutions in France or abroad, or from public or private research centers.
L'archive ouverte pluridisciplinaire HAL, est destinée au dépôt et à la diffusion de documents scientifiques de niveau recherche, publiés ou non, émanant des établissements d'enseignement et de recherche français ou étrangers, des laboratoires publics ou privés. 


\title{
Global nonlinear dynamics of thin aerofoil wakes
}

\author{
Benoît PIER and Nigel PEAKE
}

\begin{abstract}
In the present investigation of thin aerofoil wakes we compare the global nonlinear dynamics, obtained by direct numerical simulations, to the associated local instability features, derived from linear stability analyses. A given configuration depends on two control parameters: the Reynolds number Re and the adverse pressure gradient $m$ (with $m<0$ ) prevailing at the aerofoil trailing edge. Global instability is found to occur for large enough Re and $|m|$; the naturally selected frequency is determined by the local absolute frequency prevailing at the trailing edge.
\end{abstract}

\section{Introduction}

The global dynamics of spatially developing shear flows is known to closely depend on its local stability features. Previous linear stability analyses $[11,10]$ have established that thin aerofoil wakes display local absolute instability near the trailing edge. In the present investigation we revisit this flow and examine its fully nonlinear régime within the framework of nonlinear global mode theory $[9,4]$.

With $x$ and $y$ denoting streamwise and cross-stream coordinates respectively, we consider the semi-infinite flow domain $(x>0,-\infty<y<+\infty)$ corresponding to the region downstream of the aerofoil trailing edge, where a symmetric velocity profile is imposed, represented by the double FalknerSkan boundary layer solutions with negative pressure gradient $m$. The pa-

Benoît PIER

Laboratoire de mécanique des fluides et d'acoustique (CNRS - Université de Lyon)

École centrale de Lyon, 36 avenue Guy-de-Collongue, 69134 Écully cedex, France.

Nigel PEAKE

Department of Applied Mathematics and Theoretical Physics, University of Cambridge, Centre for Mathematical Sciences, Wilberforce Road, Cambridge, CB3 OWA, UK. 
rameter $m$ is varied from $m=0$ (double-Blasius wake) to $m=-0.09$ (near flow separation); Reynolds numbers from $\mathrm{Re}=50$ up to $\mathrm{Re}=5000$ are considered. The entire study is carried out for two-dimensional incompressible flows governed by the Navier-Stokes equations.

\section{Global nonlinear dynamics obtained by direct numerical simulations}

Global flow dynamics are obtained by direct numerical simulation of the governing equations, implementing a method similar to that used in [8]. Timeintegration is performed via a fractional step method of second-order accuracy in time. Spatial discretization combines fifth-order finite differences in the $x$-direction and Chebyshev collocation points in the $y$-direction. The streamwise grid is uniformly stretched in the downstream direction and the collocation points are mapped onto the entire cross-stream domain via an algebraic transformation. Non-reflecting boundary conditions [5] are used at the downstream boundary of the computational domain.

At low values of Re the wake is globally stable and the system converges towards a steady $y$-symmetric solution of the Navier-Stokes equations. Above a critical Reynolds number, which depends on the value of the parameter $m$, global instability occurs, and time-dependent fluctuations develop in the wake. After a transient régime, a fully developed self-sustained downstreampropagating vortex-street is observed. Figure 1 shows snapshots of streamwise and cross-stream velocity fields of such a saturated régime obtained with $\mathrm{Re}=5000$ and $m=-0.085$.

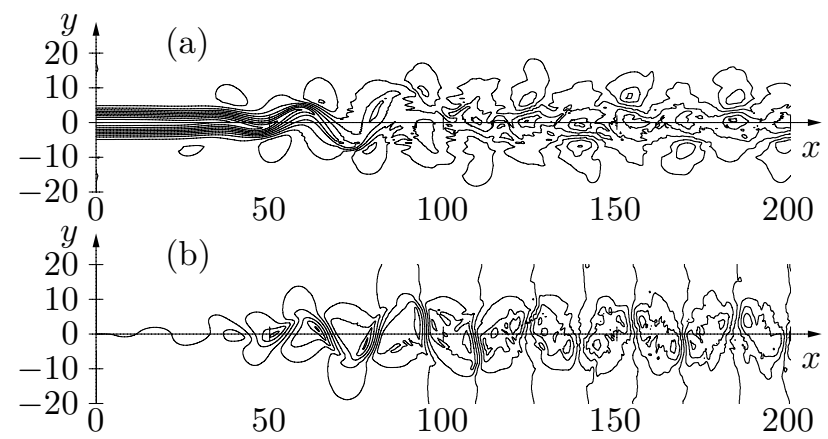

Fig. 1 Snapshot of fully developed nonlinear régime at Re $=5000$ and $m=-0.085$. Equispaced isocontours of (a) streamwise and (b) cross-stream velocity fields.

The naturally selected vortex-shedding frequencies are obtained by recording time-series at different locations and computing the associated frequency spectra. These spectra display a wide range of harmonics but obtaining them at different spatial locations demonstrates that the entire flow is tuned to 
a single fundamental frequency. The dependence of the selected global frequency on both control parameters is given in figure 2. From these curves it is concluded that, for given $m$, the global frequency is nearly independent of Re, except near onset. However, the global frequency significantly depends on $m$ : the vortex shedding slows down when $m$ is decreased and separation of the inlet velocity profile is approached.

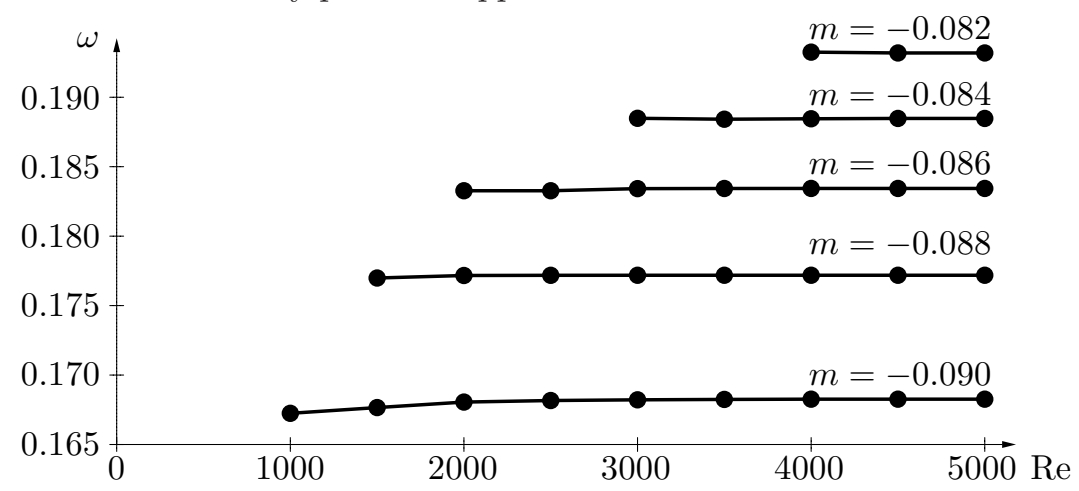

Fig. 2 Reynolds number dependence of the naturally selected global frequencies for different values of the parameter $m$.

\section{Basic flow and local absolute frequencies}

This section investigates the unperturbed basic flow and its local stability features, covering the same parameter ranges as the previous section.

The basic flow is, by definition, a steady solution of the Navier-Stokes equations. Basic flow fields may be computed by imposing a symmetry condition along the $x$-axis and considering only the domain $y \geq 0$. At very large Reynolds numbers, however, this symmetry condition is not enough to stabilize the basic flow, and the selective frequency damping method [1] has therefore also been implemented. Thus, the basic flows are readily obtained by numerical integration of the governing equations, using the same code as in the previous section with only minor modifications.

For given values of Re and $m$, local stability characteristics of the associated basic flow are then derived at a given streamwise station by freezing the $x$-coordinate and studying the equivalent parallel shear flow of corresponding velocity profile. Such an approach can be justified by rigorous asymptotic analyses based on the assumption of slow streamwise development of the basic flow. In the case of thin aerofoil wakes, this is certainly a reasonable assumption, except very close to the trailing edge; and even in the very near wake region, the assumption of weak spatial inhomogeneity only breaks down for small values of both Re and $|m|$. 
Local linear instability waves developing on these velocity profiles are then governed by the Orr-Sommerfeld equation, which yields the local linear dispersion relation

$$
\omega=\Omega(k ; x ; \operatorname{Re}, m)
$$

between the complex frequency $\omega$ and complex wavenumber $k$ at streamwise station $x$ for the basic flow characterized by Re and $m$.

In the context of open shear flows, a crucial feature is the complex absolute frequency defined for parallel flows as the frequency observed at a fixed spatial location in the long-time linear response to an initial impulse. The local absolute frequency $\omega_{0}(x ; \operatorname{Re}, m)$ is derived in classical fashion by applying a pinch-point criterion $[3,2]$, equivalent to a vanishing group velocity condition

$$
\omega_{0}(x ; \operatorname{Re}, m)=\Omega\left(k_{0} ; x ; \operatorname{Re}, m\right) \quad \text { with } \quad \frac{\partial \Omega}{\partial k}\left(k_{0} ; x ; \operatorname{Re}, m\right)=0 .
$$

Figure 3 illustrates the streamwise evolution of the local absolute growth rate $\omega_{0, i}$ and real absolute frequency $\omega_{0, r}$. It is seen that the largest absolute growth rate $\omega_{0, i}$ occurs near the trailing edge at $x=0$. The growth rate increases with (adverse) pressure gradient $|m|$, and the size of the absolutely unstable domain (where $\omega_{0, i}>0$ ) increases with Reynolds number. All basic wake flows of this investigation are at least marginally absolutely unstable at $x=0$. However, for moderate values of $m$, the extent of the absolutely unstable domain is rather limited. It is only for stronger adverse pressure gradients, close to detachment, that relatively strong absolute instability is obtained.
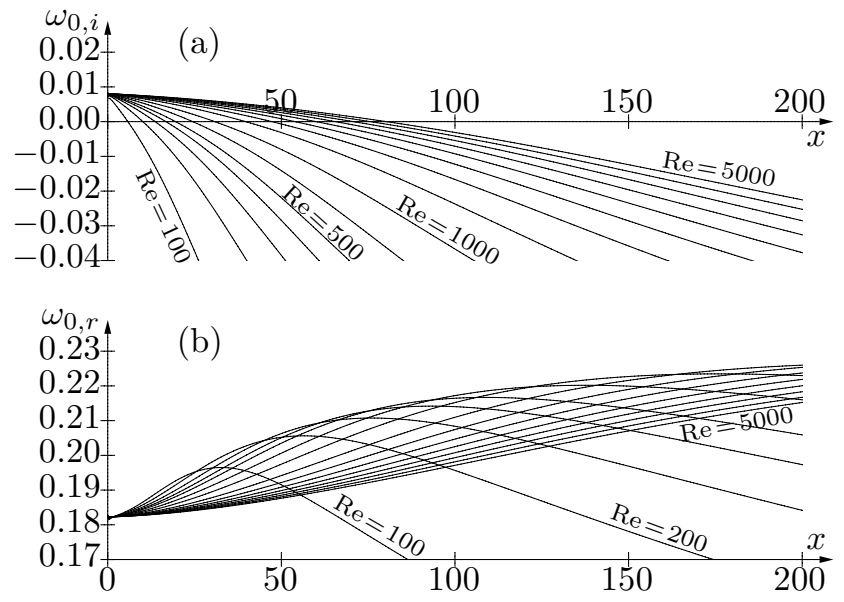

Fig. 3 Streamwise variation of (a) imaginary and (b) real parts of local absolute frequencies obtained with $m=-0.085$ and $\mathrm{Re}=100,200,300,400,500,700,1000,1500, \ldots$, 5000 . 


\section{Discussion}

The spatial structure of the global mode is the following. The upstream region, close to the trailing edge, is covered by small-amplitude perturbations that propagate downstream. While propagating downstream, their amplitude grows exponentially until nonlinear saturation occurs. Further downstream, a finite-amplitude wavetrain prevails, the amplitude and wavenumber of which slowly vary with downstream distance. In the far wake, the wave amplitude slowly decays while small-scale features may develop for high-Reynoldsnumber configurations.

A comparison of the vortex shedding frequency measured by DNS and the real part of the absolute frequency $\omega_{0, r}(x=0)$, prevailing at the trailing edge, is shown in figure 4 . This plot shows that the naturally selected frequency is in excellent agreement with the absolute frequency prevailing at the trailingedge, thereby demonstrating that the global dynamics of thin aerofoil wakes is closely linked to the local absolute stability characteristics of the associated base flow.

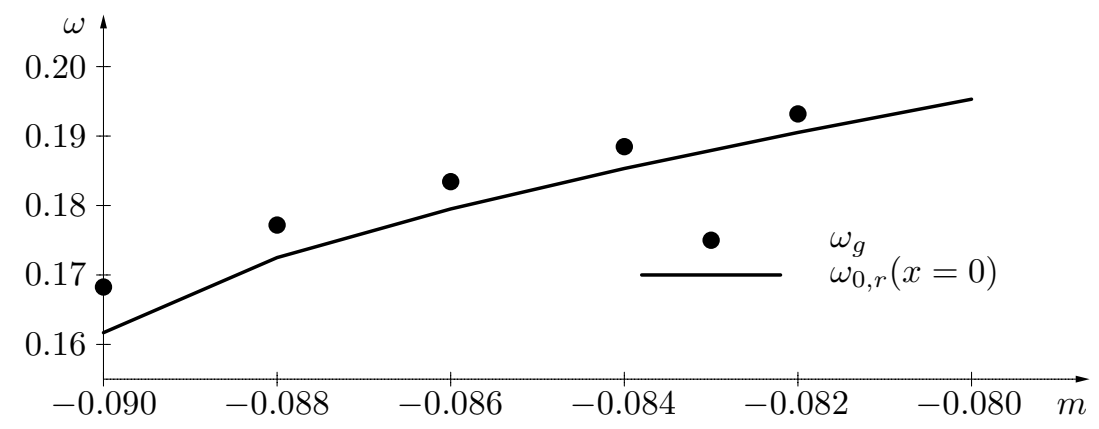

Fig. 4 Comparison of measured vortex shedding frequency $\omega_{g}$ and trailing-edge absolute frequency $\omega_{0, r}(x=0)$.

A nonlinear saturation station $x_{\text {sat }}$ may be defined as the streamwise position where the envelope of the global mode reaches $90 \%$ of its maximum. Figure 5 shows how the thus computed $x_{\text {sat }}$ depends on Re for selected values of $m$. The saturation length appears to converge towards a constant value for Re $\rightarrow \infty$ while it would diverge at the critical Reynolds number for onset of global instability. A somewhat intriguing result is also derived from this plot: the nonlinear saturation may occur downstream of the absolutely unstable domain. It has been verified that this observation does not depend on the length of the computational domain. Nevertheless, we are currently implementing a completely different numerical method to verify the robustness of this counterintuitive result.

We are also studying the controllability of this flow by applying smallamplitude harmonic forcing near the trailing edge. Preliminary results in- 


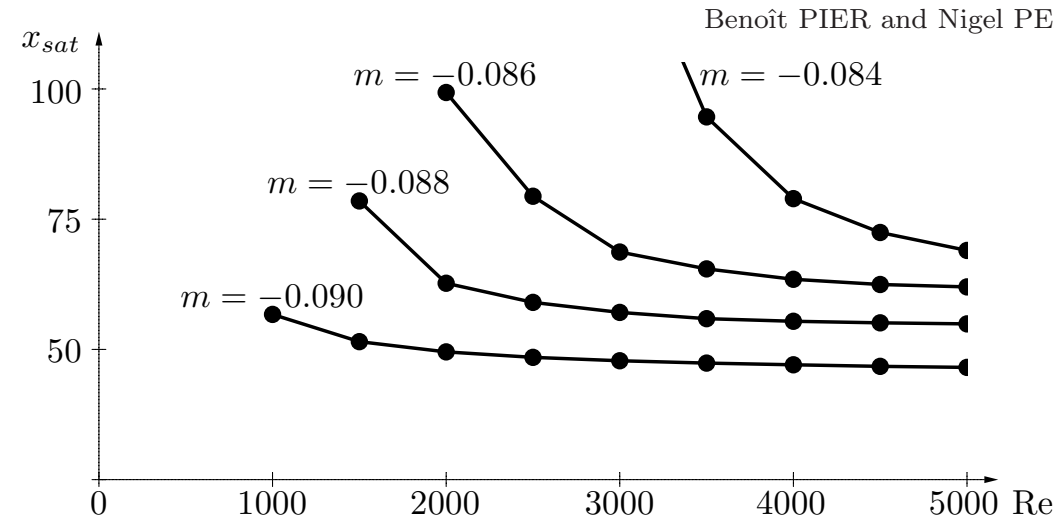

Fig. 5 Variation of nonlinear saturation station with control parameters Re and $m$.

dicate that the open-loop control technique developed in a different context $[6,7]$ is capable of modifying the present flow.

Computing resources from Fédération lyonnaise de calcul hautes performances (FLCHP) and Institut national de physique nucléaire et de physique des particules (IN2P3) are gratefully acknowledged.

\section{References}

1. Åkervik, E., Brandt, L., Henningson, D.S., Hœpffner, J., Maxen, O., Schlatter, P.: Steady solutions of the Navier-Stokes equations by selective frequency damping. Phys. Fluids 18, 068102 (2006)

2. Bers, A.: Space-time evolution of plasma instabilities - absolute and convective. In: M. Rosenbluth, R. Sagdeev (eds.) Handbook of plasma physics, pp. 451-517. NorthHolland, Amsterdam (1983)

3. Briggs, R.J.: Electron-stream interaction with plasmas. M.I.T. Press, Cambridge, Mass. (1964)

4. Chomaz, J.M.: Fully nonlinear dynamics of parallel wakes. J. Fluid Mech. 495, 57-75 (2003)

5. Jin, G., Braza, M.: A nonreflecting outlet boundary condition for incompressible unsteady Navier-Stokes calculations. J. Comp. Phys. 107, 239-253 (1993)

6. Pier, B.: Open-loop control of absolutely unstable domains. Proc. R. Soc. Lond. A 459, 1105-1115 (2003)

7. Pier, B.: Primary crossflow vortices, secondary absolute instabilities and their control in the rotating-disk boundary layer. J. Eng. Math. 57, 237-251 (2007)

8. Pier, B.: Local and global instabilities in the wake of a sphere. J. Fluid Mech. 603, 39-61 (2008)

9. Pier, B., Huerre, P., Chomaz, J.M.: Bifurcation to fully nonlinear synchronized structures in slowly varying media. Physica D 148, 49-96 (2001)

10. Taylor, M.J., Peake, N.: A note on the absolute instability of wakes. Eur. J. Mech. B/Fluids 18, 573-579 (1999)

11. Woodley, B.M., Peake, N.: Global linear stability analysis of thin aerofoil wakes. J. Fluid Mech. 339, 239-260 (1997) 